\title{
Towards improving tsetse fly paratransgenesis: stable colonization of Glossina morsitans morsitans with genetically modified Sodalis
}

\author{
Linda De Vooght*, Severien Van Keer and Jan Van Den Abbeele*
}

\begin{abstract}
Background: Tsetse flies (Glossina sp.) refractory to trypanosome infection are currently being explored as potential tools to contribute in the control of human and animal African trypanosomiasis. One approach to disrupt trypanosome transmission by the tsetse fly vector involves the use of paratransgenesis, a technique that aims to reduce vector competence of disease vectors via genetic modification of their microbiota. An important prerequisite for developing paratransgenic tsetse flies is the stable repopulation of tsetse flies and their progeny with its genetically modified Sodalis symbiont without interfering with host fitness.

Results: In this study, we assessed by qPCR analysis the ability of a chromosomally GFP-tagged Sodalis (recSodalis) strain to efficiently colonize various tsetse tissues and its transmission to the next generation of offspring using different introduction approaches. When introduced in the adult stage of the fly via thoracic microinjection, recSodalis is maintained at high densities for at least 21 days. However, no vertical transmission to the offspring was observed. Oral administration of recSodalis did not lead to the colonization of either adult flies or their offspring. Finally, introduction of recSodalis via microinjection of third-instar larvae resulted in stably colonized adult tsetse flies. Moreover, the subsequent generations of offspring were also efficiently colonized with recSodalis. We show that proper colonization of the female reproductive tissues by recSodalis is an important determinant for vertical transmission.
\end{abstract}

Conclusions: Intralarval microinjection of recSodalis proves to be essential to achieve optimal colonization of flies with genetically modified Sodalis and its subsequent dissemination into the following generations of progeny. This study provides the proof-of-concept that Sodalis can be used to drive expression of exogenous transgenes in Glossina morsitans morsitans colonies representing a valuable contribution to the development of a paratransgenic tsetse fly based control strategy.

Keywords: Paratransgenesis, Glossina, Sodalis glossinidius, Colonization, Transmission, GFP

\section{Background}

As vector-borne diseases continue to present significant threats to human, animal and plant health, there is a perpetual need to improve existing and develop new control strategies. Vector control has always been and still remains a key component of any integrated program to control vector-borne diseases. In the light of increasing

\footnotetext{
* Correspondence: Idevooght@itg.be; jvdabbeele@itg.be Department of Biomedical Sciences, Unit of Veterinary Protozoology, Institute of Tropical Medicine Antwerp, Antwerp, Belgium
}

insecticide resistance combined with the growing awareness of the negative environmental and ecological consequences caused by the widespread use of insecticides, genetic control of the insect vector provides a valuable addition to the armory of tools already available.

Tsetse flies (Glossina sp.) are medically and agriculturally important vectors that transmit Trypanosoma spp. parasites responsible for human sleeping sickness and animal African trypanosomiasis (AAT). Today, prevention and control programs are mainly based on elimination of 
the parasite reservoir and vector-oriented control since there are no prophylactic drugs or vaccines available and the few available treatments present serious side effects [1, 2]. The generation of a trypanosome-resistant tsetse fly that is incapable of transmitting the trypanosome parasite would be extremely valuable in integrated control programs against African Trypanosomiasis by complementing the sterile insect technique (SIT) that has proven effective in eradicating tsetse in isolated pockets [3]. At present, SIT relies on the massive release of sterile male tsetse flies accompanied by a temporary increase in the number of potential vectors for trypanosomes, especially during the first few years of the control campaign. As such, the use of tsetse flies with a refractory phenotype would render this approach less controversial especially when applied in areas where human sleeping sickness is also occurring beside AAT.

Since tsetse flies are not amenable to germ-line transformation due to their viviparous reproductive biology, a paratransgenic approach using the tsetse fly secondary symbiont Sodalis glossinidius as a delivery system for anti-trypanosomal components is currently of considerable interest to achieve a refractory phenotype. Sodalis glossinidius is a maternally inherited gram-negative bacterial endosymbiont of the tsetse fly that can be found both inter- and intracellularly in the tsetse fly midgut, muscle, fat body, milk glands, and salivary glands [4]. A crucial step in developing paratransgenic tsetse is the stable colonization of flies and their progeny with recombinant Sodalis strains expressing trypanosome-interfering proteins in insect tissues where trypanosome parasites reside. The current method to introduce recombinant Sodalis into the tsetse fly relies on thoracic microinjection into the haemolymph [5].

Recently, we developed a plasmid-based expression system allowing Sodalis to constitutively express and release functional trypanosome-binding nanobodies (Nbs) targeting the Trypanosoma brucei VSG, in an in vitro culture system as well as in vivo in different tissues of the tsetse fly following introduction via thoracic microinjection [6, 7]. However, our experiments showed that when introducing the plasmid-bearing Sodalis into adult tsetse flies by intrathoracic injection, transmission to the progeny is limited, hampering the establishment of a paratransgenic tsetse fly colony. This inefficient transfer upon injection in the adult female fly could be attributed to plasmid loss by the recombinant Sodalis and/or its inability to efficiently colonize the female milk glands which is a prerequisite for recombinant Sodalis transmission to the intra-uterine larvae through the maternal milk secretion. To overcome the need for continuous selection pressure inherent to plasmid-based gene expression, we recently developed a Tn7-based transposition system for the stable integration of transgenes into a transcriptional highly active region of the Sodalis genome [8].

In this study, we investigated alternative approaches to stably colonize tsetse flies and their subsequent generations with recombinant Sodalis using a genetically engineered Sodalis strain constitutively expressing green fluorescent protein (GFP) for the proof-of-concept. First we assessed the suitability of Sodalis native and non-native promoters for the chromosomal expression of GFP using flow cytometry. Next we evaluated the ability of GFP-tagged Sodalis (recSodalis) to efficiently colonize various tsetse tissues after being introduced in the fly via two different approaches; i) introduction in wild type (WT) Sodalis cleared adult female flies either via thoracic microinjection or blood meal supplementation and ii) microinjection of third-instar larvae. Besides colonization of the $F_{0}$ generation, vertical transmission to and colonization of the progeny by the recSodalis strain along multiple generations was investigated. We demonstrate that intralarval microinjection of recSodalis proves to be essential to achieve maternal transfer and subsequent establishment of a genetically modified Sodalis strain into the following generations of progeny.

\section{Results}

Chromosomal expression of a reporter gene controlled by a native or a heterologous constitutive promoter We assessed the capability of two different promotors i.e., the E. coli lac $\mathrm{Z}$ and the native Sodalis glossinidius groEL promoter, to chromosomally express GFP in Sodalis in in vitro culture conditions as well as in vivo. Since Sodalis is devoid of a lac repressor, the presence of the E. coli lac promoter results in a constitutive expression of the transgene. The universal heat shock chaperonin GroEL is constitutively expressed at high levels in Sodalis by its efficient promoter. Both the E. coli lacZ as well as the Sodalis GroEL promotor proved to be functional for the expression of active GFP in in vitro conditions as well as in vivo in the haemolymph. Flow cytometry analysis showed that chromosomal GFP expression under control of the lac $\mathrm{Z}$ promotor resulted in higher expression levels in vitro as well as in vivo compared to the GroEL promotor (Fig. 1). Since promoters that drive the strong expression of effector genes in transgenic insects are likely to result in more abundant transcription and increased effectiveness, the lacZ promotor was used for all future experiments involving the expression of effector genes in Sodalis.

Recombinant symbiont introduction into adult tsetse flies From our previous studies we know that transient treatment of G. morsitans morsitans flies with streptozotocin reduces the WT Sodalis population which is crucial for allowing recombinant Sodalis to proliferate inside its 

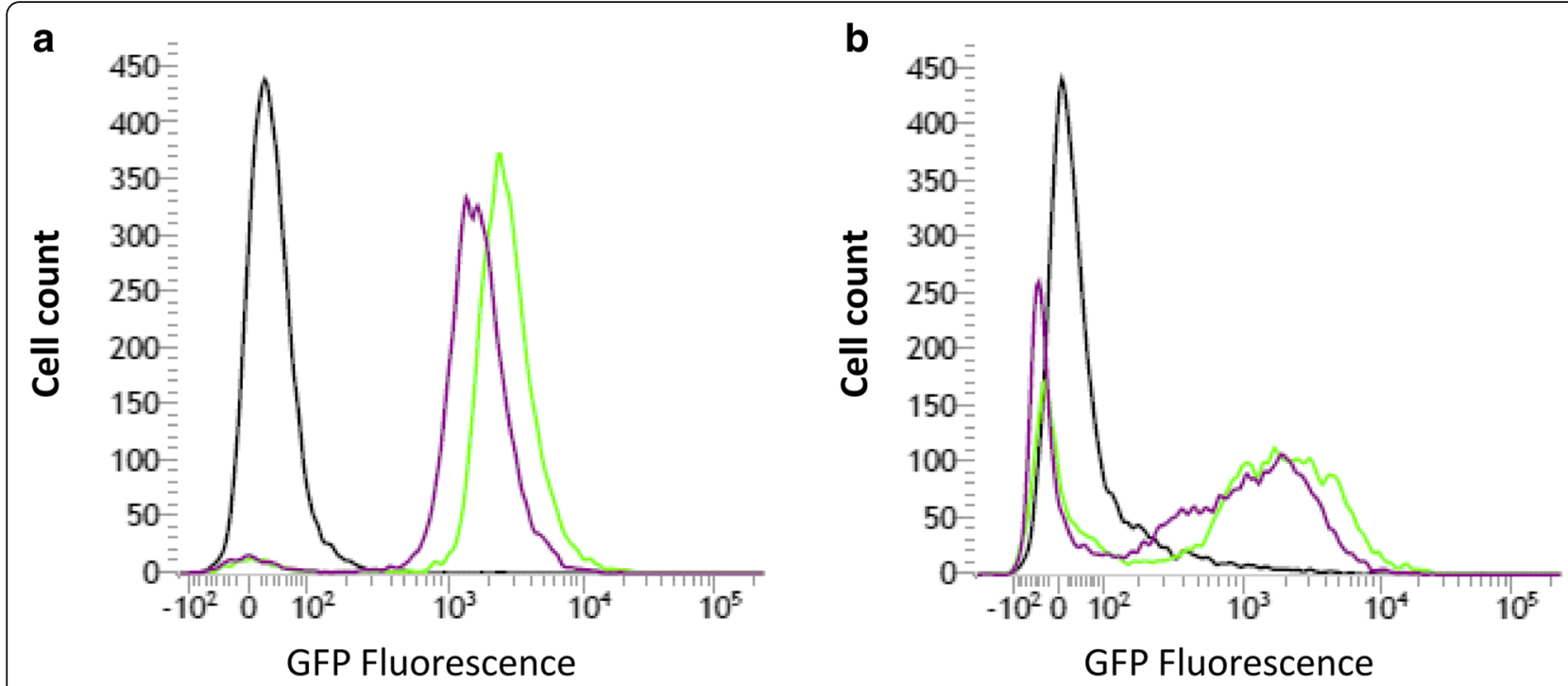

Fig. 1 a FACS plot overlay showing in vitro green fluorescent protein (GFP) expression of WT Sodalis cells (black histogram), chromosomally GFPtagged Sodalis cells harboring the E. coli lacZ promotor (green histogram) and chromosomally GFP-tagged Sodalis cells harboring the Sodalis GroEl promotor (purple histogram); b GFP expression of Sodalis in WT flies (black histogram) and flies injected with chromosomally GFP-tagged Sodalis cells harboring the E. coli lacZ promotor (green histogram) and the Sodalis GroEl promotor (purple histogram). The low intensity peaks result from the native WT Sodalis population in injected flies

host without affecting the obligatory Wigglesworthia symbiont population [7].

Prior to the introduction of recSodalis, female flies received 3 streptozotocin $(20 \mu \mathrm{g} / \mathrm{ml})$ supplemented blood meals reducing the WT Sodalis population by 98 , 97 and $93 \%$ in abdomen, thorax and female reproductive organs respectively, compared to flies fed on normal blood (Additional file 1). Next, we analyzed the ability of recSodalis to colonize different tissues of streptozotocin-treated female tsetse flies and its transmission to the progeny after introduction via either thoracic microinjection, blood meal supplementation or a combination of the two. This analysis was done by
qPCR based estimation of the amount of recSodalis CFU present in abdomen, thorax and reproductive organs over a 21 day period.

RecSodalis was able to proliferate in abdomen and thorax tissues of flies injected intrathoracically with $5 \times$ $10^{6}$ recSodalis CFU and remained present at high densities throughout the course of the 21-day observation period (Fig. 2a). A similar colonization pattern was observed in flies receiving both a thoracic injection and a recSodalis supplemented blood meal with recSodalis densities persisting at $10^{5}$ and $10^{6} \mathrm{CFU}$ (DNA equivalent) in abdomen and thorax tissues respectively throughout the 21-day observation period (Fig. 2b). For

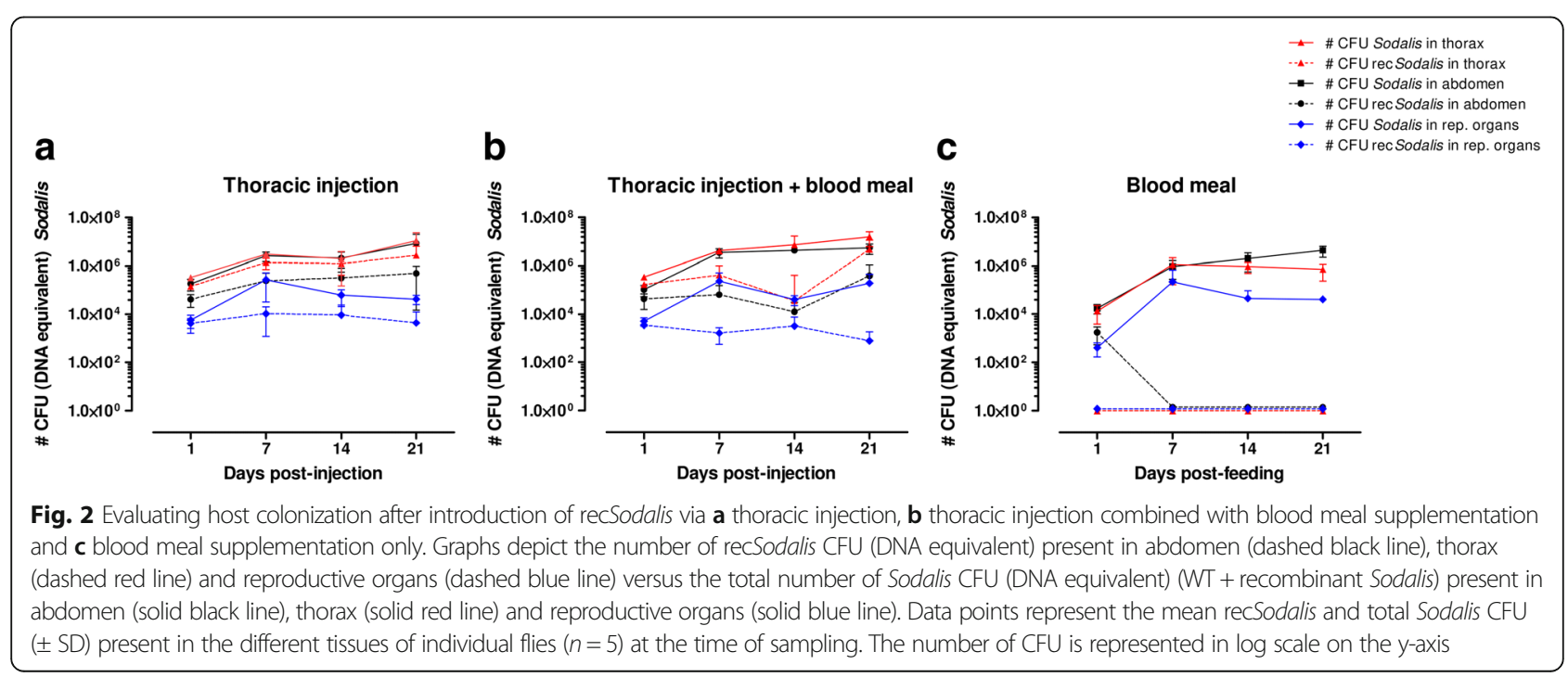


both conditions, recSodalis could be detected in the female reproductive organs, however colonization was limited with less than $10 \%$ of the total Sodalis population proving to be recombinant (Fig. $2 \mathrm{a}$ and b). RecSodalis offered to flies via only blood meal supplementation was not able to maintain itself in the fly: $24 \mathrm{~h}$ post-feeding recSodalis could not be detected in either abdomen, thorax or reproductive tissues (Fig. 2c).

The streptozotocin treatment had a negative effect upon tsetse fecundity; in all experimental groups an arrest in the reproductive cycle of the females was seen for $\sim 21$ days, after which only a proportion of the experimental female flies that received recSodalis via thoracic injection combined with blood meal supplementation and blood meal supplementation only started to produce offspring again (respectively $50 \%$ and $46 \%$ of the female flies). qPCR showed that streptozotocin had no effect on Wigglesworthia, the primary endosymbiont of tsetse known to be important for fertility. Upon dissection of the reproductive organs of sterile females we did observe atrophy of the ovaries suggesting that the observed reduction in fecundity resulted from a more direct effect on the host rather than on its primary symbiont.

Finally, transmission dynamics of the recombinant bacteria to the progeny were also evaluated by qPCR; no vertical transmission was observed as recSodalis could not be detected in the F1 generation of any of the mother flies that received recSodalis via intrathoracic injection or feeding (Fig. 3).

\section{Recombinant symbiont introduction into third-instar larvae}

Third-instar larvae, collected immediately after larviposition were injected with $10^{6} \mathrm{CFU}$ of recSodalis. Pupated larvae were allowed to hatch and the recombinant as well as the total Sodalis densities present in abdomen and thorax tissues from the emerged male adult flies $\left(F_{0}\right)$ were measured using qPCR. Emerged female flies were mainly kept for mating to assess the vertical transmission of recSodalis to the offspring, therefore colonization of recSodalis in female reproductive organs and midgut was only assessed on day 48 post-eclosion. The abdomen and thorax from male flies that emerged from larvae $\left(\mathrm{F}_{0}\right)$ injected with $1 \times 10^{6} \mathrm{CFU}$ (Fig. 4a) were efficiently colonized by recSodalis from day 1 post-emergence with the population growing to peak densities of around $4 \times 10^{6} \mathrm{CFU}$ (DNA equivalent) on day 14 in both abdomen and thorax tissues. The reproductive organs of female flies emerged from injected larva were efficiently colonized with $87 \%$ of the total

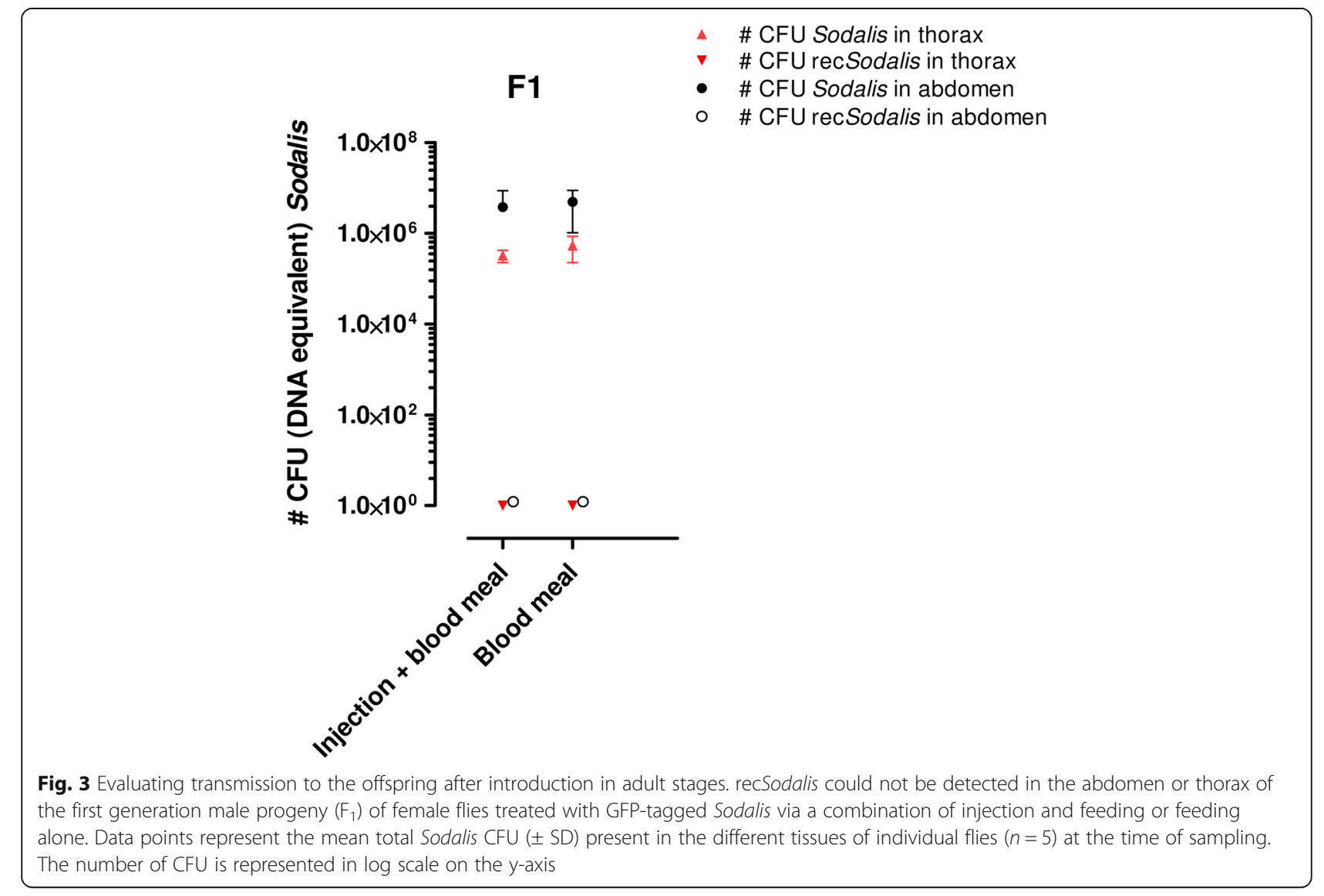



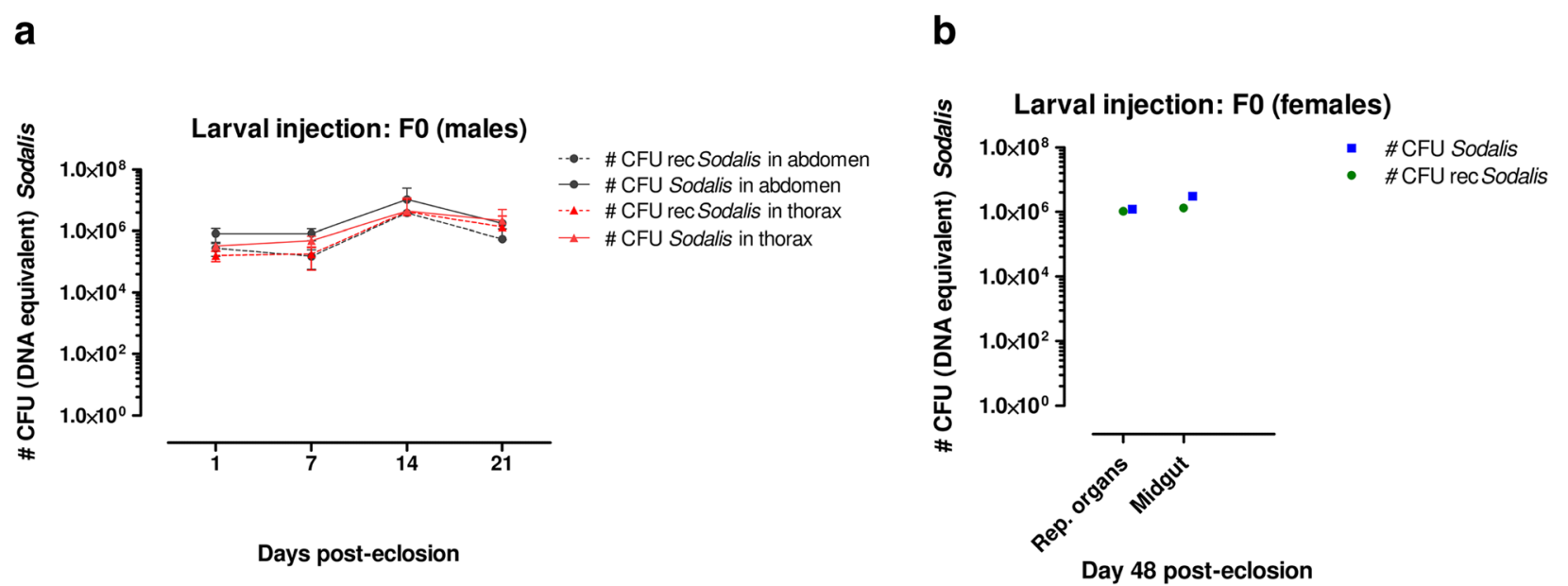

Fig. 4 Evaluating host colonization after introduction of recSodalis via larval injection. a the number of recSodalis CFU (DNA equivalent) present in abdomen (dashed black line) and thorax (dashed red line) tissues of male flies emerged from larvae injected with $1 \times 10^{6}$ recombinant CFU versus the total number of Sodalis CFU DNA equivalent (WT + recombinant Sodalis) present in abdomen (solid black line) and thorax tissues (solid red line). $\mathbf{b}$ the number of recSodalis CFU (DNA equivalent) present in female reproductive tissues and midguts (green ) versus the total number of Sodalis CFU DNA equivalent (WT + recombinant Sodalis) (blue- ). Data points represent the mean recSodalis and total Sodalis CFU ( \pm SD) present in the different tissues of individual flies $(n=5)$ at the time of sampling. The number of CFU is represented in log scale on the $y$-axis

Sodalis population being recombinant, reaching a $10^{3}$-fold higher density compared to the thoracic introduction method (Fig. 4b).

Furthermore, recSodalis was transmitted to both male and female offspring resulting from female flies microinjected at the larval stage (Fig. 5). We detected recSodalis to be present in densities comparable to natural Sodalis levels present in WT flies in all tissues including abdomen, thorax, female reproductive tissues and midgut. We continued to breed from the offspring until the F3 generation (around 150 days after the initial $F_{0}$ larvae injection) and we observed that all flies remained highly positive for recSodalis.

\section{Discussion}

Despite the interest in Sodalis as an in vivo drug delivery vehicle to control trypanosome parasite development in the tsetse fly, significant hurdles still need to be overcome towards achieving this goal. The stable colonization of adult flies and their progeny with the introduced genetically modified Sodalis strain during multiple generations is an important prerequisite for Sodalis

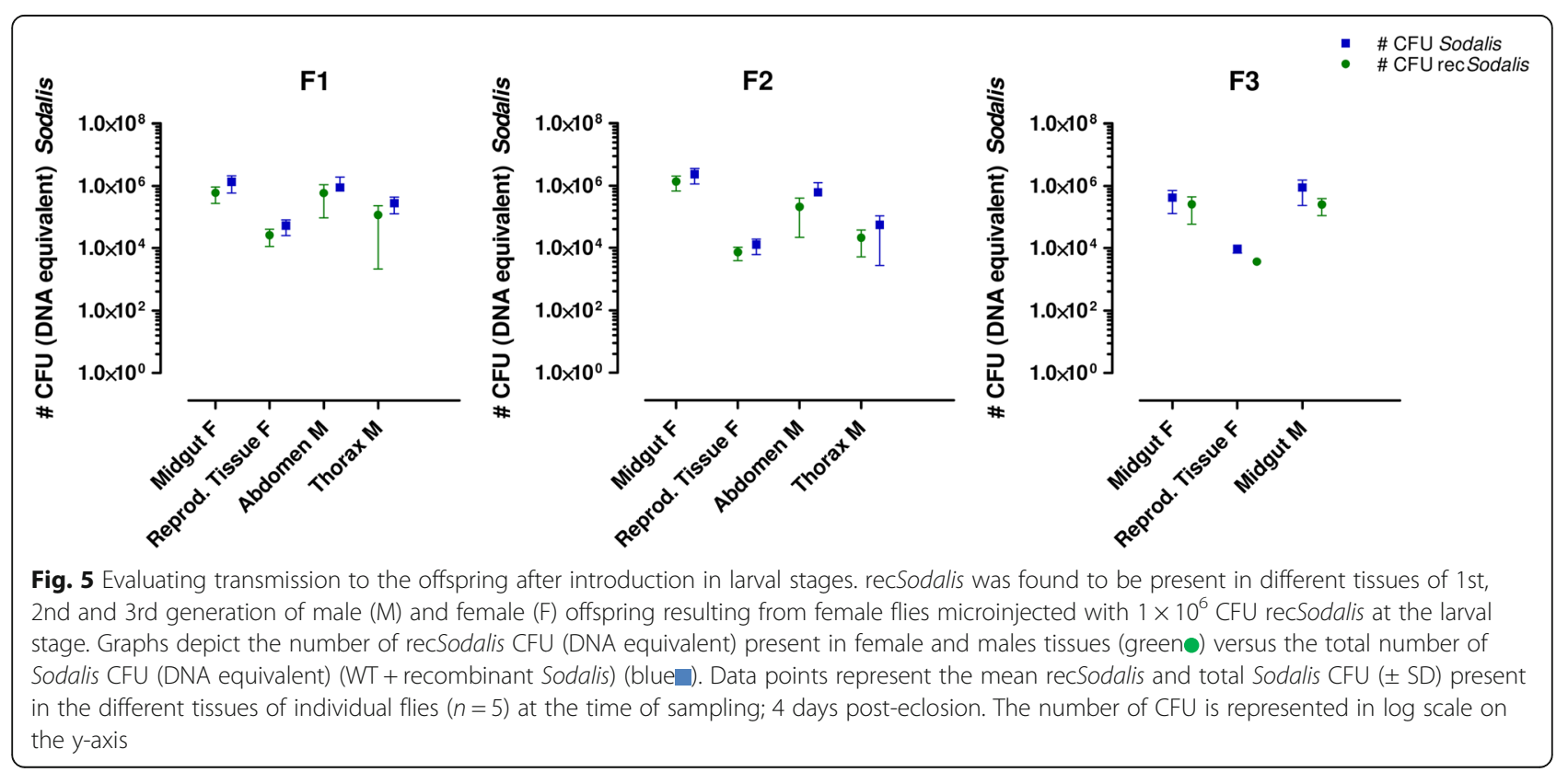


to be used as a paratransgenic platform. Therefore, in this study we aimed to optimize the existing Sodalis re-introduction methods and evaluate the colonization efficiencies of the re-introduced bacteria in the tsetse fly along subsequent generations. Using qPCR analysis we assessed the ability of a chromosomally GFP-tagged Sodalis (recSodalis) strain to efficiently colonize various tsetse tissues after being introduced in the tsetse fly via two different approaches; i) introduction in WT Sodalis cleared adult female flies either via thoracic microinjection or blood meal supplementation and ii) introduction in third-instar larvae. Besides colonization of the $\mathrm{F}_{0}$ generation, vertical transmission to and colonization of the progeny by the recSodalis strain was also investigated.

When introduced via thoracic microinjection in the haemolymph of adult flies cleared of WT Sodalis, recSodalis was able to establish and proliferate in both abdomen and thorax of injected flies. However, the recSodalis population predominantly remained present locally at the site of injection i.e. in the haemolymph. Indeed, the lower number of recSodalis observed in female reproductive organs indicates limited dissemination of recSodalis to these tissues via the haemolymphatic circulation of the fly. We next investigated an alternative, oral route of recombinant symbiont introduction in adult tsetse. Maltz et al. [9] were able to efficiently colonize tsetse fly gut tissue with mutant Sodalis via a complement inactivated blood meal, supplemented with $500 \mathrm{CFU} / \mathrm{ml}$ Sodalis. However, all attempts to colonize tsetse fly tissues by this oral administration route were not successful in our study.

Both the thoracic microinjection and oral introduction routes of recSodalis in adult females did not result in the vertical transmission of the bacterium to the offspring. From the above described experiments where recSodalis is introduced in adult female flies we can conclude that recSodalis predominantly remains present at the site of injection with little dissemination to the reproductive organs and no vertical transfer to the offspring. This suggests that the ability of the recSodalis bacterium to reach and colonize the reproductive organs (milk glands, ovarial tissue) is compromised in the adult fly stage. Unlike many other insects, tsetse flies display a viviparous mode of reproduction where the female fly gives birth to a full grown (third-instar) larva that has been nourished within the female uterus by maternal milk gland secretions containing essential nutrients for larval development as well as the fly's symbionts Wigglesworthia and Sodalis. In the milk gland, Sodalis resides both extracellularly in the lumen as intracellularly within the milk gland secretory cells [10]. Cell invasion has been shown to play an important factor for Sodalis to be vertically transmitted to the offspring. In a mutant line of Sodalis, lacking a crucial type III secretion system invasion gene $(i n \nu C)$, establishment of Sodalis infections in tsetse progeny was compromised, thus indicating its role in transmission to progeny [11]. Furthermore, previous studies have demonstrated that Sodalis motility genes, fliC and $m o t A$, and cell invasion genes, invA1 and $\operatorname{inv} A 2$, are up regulated in the larval and early pupal stages, and not in adult tsetse flies [12].

These studies led us to hypothesize that only at the larval stages Sodalis motility and invasion capabilities are activated and that these are essential factors to achieve an efficient colonization of the tsetse fly reproductive tissues and subsequent transmission to the offspring. This can be anticipated by introducing recSodalis during the new-born larval stage. Indeed, our data showed that recSodalis introduction via intralarval microinjection of third-instar larvae results in stably colonized adult tsetse flies. Moreover, midgut and female reproductive organs were efficiently colonized and recSodalis was stably transmitted throughout the subsequent progeny generations (evaluated until the 3rd generation in this study which corresponds to a total time period of around 150 days after the $\mathrm{F}_{0}$ larval injection). These results generate new questions regarding the biology of the factors that promote Sodalis colonization of tissues in the developing larva and their vertical transmission demanding further study.

\section{Conclusions}

Collectively, this study demonstrates that recSodalis introduced via larval injection can be used to drive expression of exogenous transgenes in G. m. morsitans and that the recombinant bacteria can be transmitted to subsequent generations, similar to wild-type bacteria. This implies that a tsetse fly colony can be established with flies harboring a modified Sodalis of interest. This is a crucial step forward as it is an essential requirement for the mass rearing of paratransgenic tsetse flies as part of the SIT technique to control African trypanosomiasis in the field.

\section{Methods}

\section{Insects, bacterial strains and culture conditions}

Glossina morsitans morsitans (Westwood) from the colony at the Institute of Tropical Medicine Antwerp (ITM), that originated from pupae collected in Kariba (Zimbabwe) and Handeni (Tanzania), were used in all experiments. Flies, maintained at $26^{\circ} \mathrm{C}$ and $65 \%$ relative humidity, were fed 3 days per week with defibrinated bovine blood (Slovak Academy of Sciences (SAS), Bratislava) using an artificial membrane system. Sodalis glossinidius strains used in this study were isolated from the hemolymph of surface-sterilized flies from the ITM colony. The generation of the GFP-tagged Sodalis strain has already been described [8] and was based on 
Tn7-mediated site-specific transposition. Briefly, plasmid pGRG25 [13] featuring the tnsABCD genes expressed under control of the pBAD promoter and a multiple cloning site, flanked by the left and right ends of Tn7 was used for transgene insertion into the chromosome of S. glossinidius. The $g f p$ gene in fusion with either the lacZ or GroEl promotor was cloned into NotI-XhoI of the pGRG25 multiple cloning site, located between the Tn7R and Tn7L end sequences, at which the transposition proteins act to move the element. The plasmids were transformed into WT S. glossinidius cells using a heat shock method. Transformed cells were allowed to recover overnight at $26{ }^{\circ} \mathrm{C}$ prior to plating onto Mitsuhashi-Maramorosch (MM; PromoCell) -blood agar, supplemented with ampicillin. A single recombinant $S$. glossinidius colony was then inoculated into liquid culture and grown in the presence of arabinose allowing TnsABCD to be expressed leading to transgene insertion. Transposition was verified by PCR using primers that flank the attTn7 site (GlmS_Fw: 5' -TATGAAG ATTATTCCCCTGCCGCA-3'; PhoS_rev: 5' - CCATTT AGCGTAAACCGGCG-3') and sequencing of the PCR product. The pGRG25 plasmid was cured from recombinant Sodalis by multiple passages in the absence of ampicillin. Cultures were maintained in vitro at $26{ }^{\circ} \mathrm{C}$ in liquid MM insect medium supplemented with $10 \%(\mathrm{v} / \mathrm{v})$ heat-inactivated fetal bovine serum (FBS).

\section{FACS analysis}

The fluorescence intensity of the different chromosomally GFP-tagged Sodalis strains was measured using a FACSVerse flow cytometer (laser $488 \mathrm{~nm}$ (filter 527/32 with 507 LP filter)) (BD Biosciences) and data analysed using the BD FACSuite software package (version 1.0.5.3841). i) In vitro conditions; cells were washed once and resuspended in cold PBS at a final concentration of $5 \times 10^{6}$ cells per $\mathrm{ml}$. ii) In vivo; male teneral flies were briefly anaesthetized by cold shock and microinjected intrathoracically with a suspension of $5 \times 10^{6}$ recombinant and WT Sodalis CFU. Injections were performed under a binocular microscope, using a $5 \mu$ l Hamilton 75RN microsyringe with gauge 30 removable electrotapered needles. After 7 days, hemolymph was collected from injected flies by removing one front fly leg at the joint nearest to the thorax and applying gentle pressure to the distal tip of the abdomen; $2 \mu \mathrm{l}$ of hemolymph exuding from the wound was collected in $500 \mu \mathrm{l}$ PBS using a $10 \mu$ pipette..

\section{Recombinant symbiont introduction Introduction in adult stages}

Prior to recSodalis introduction, the (native) WT Sodalis population in adult experimental flies was suppressed via three subsequent blood meals supplemented with
$20 \mu \mathrm{g} / \mathrm{ml}$ streptozotocin (Sigma). Three days after the last streptozotocin supplemented blood meal, recSodalis was introduced in these flies by 2 alternative routes: i) Adult fly injection: flies were briefly anaesthetized by cold shock $\left(10 \mathrm{~min}\right.$ at $\left.4.0^{\circ} \mathrm{C}\right)$ and injected intrathoracically using a $5 \mu$ l Hamilton 75RN 34 gauge microsyringe (with removable electro-tapered needles) with $5 \times$ $10^{6} \mathrm{CFU}$ recSodalis (CFU = colony forming units). ii) Diet: flies were fed a single recSodalis supplemented blood meal. Given that a single fly ingests approximately $25 \mu \mathrm{l}$ of blood during one blood meal, blood was mixed to a concentration of $6.2 \times 10^{7} \mathrm{CFU} / \mathrm{ml}$ recSodalis. Subsequently, approximately $1.5 \times 10^{6} \mathrm{CFU}$ recSodalis were ingested by a single fly. The mammalian complement system was inactivated by heating blood for $1 \mathrm{~h}$ at $56{ }^{\circ} \mathrm{C}$.

\section{Introduction in larval stages}

Third-instar larvae, collected immediately after larviposition, were microinjected with $10^{6} \mathrm{CFU}$ of recSodalis using a $5 \mu \mathrm{l}$ Hamilton 75RN microsyringe with gauge 34 removable electrotapered needles and allowed to pupate. Injected pupae were maintained at $26{ }^{\circ} \mathrm{C}$ and $65 \%$ relative humidity until emergence 30 days later.

\section{qPCR based measuring of in vivo WT Sodalis and recSodalis densities}

We used a quantitative PCR (qPCR) method for the estimation of the number of WT Sodalis and recSodalis cells in tsetse fly tissues (abdomen, thorax and reproductive tissue) [7]. Briefly, triplicate cultures of WT Sodalis and recSodalis were serially diluted (10-fold) in PBS to yield a Sodalis density ranging from $10^{8} \mathrm{CFU} / \mathrm{ml}$ to $10^{2} \mathrm{CFU} / \mathrm{ml}$. DNA was extracted from each Sodalis dilution followed by qPCR using primers that amplify a region of the GFP gene present in recSodalis: (GFP_Fw, 5' - TGGCCAACACTTGTCACTAC-3' and GFP_Rev, $5^{\prime}$ - AGAAGGACCATGTGGTC -3') and primers that target a 120-bp region of the single-copy Sodalis glossinidius exochitinase (Qchi) gene present in both WT and recSodalis to determine the corresponding $\mathrm{Ct}$ values. Standard curves were generated by plotting these $C_{t}$ values against the corresponding log of Sodalis CFU $/ \mathrm{ml}$. This qPCR approach in combination with the Sodalis CFU standard curves allowed us to estimate the CFU (DNA equivalent) values present in the tsetse fly tissues of the different experimental fly series. For this, at various time points post-injection and post-larviposition, flies were sacrificed for genomic DNA extraction using the QIAGEN DNeasy extraction kit (QIAGEN). Dissections of abdomen, thorax, midgut and female reproductive organs were performed under a binocular microscope (Heerbrugg Switzerland), after sedation of the flies by cold shock $\left(10 \mathrm{~min}\right.$ at $\left.4.0^{\circ} \mathrm{C}\right)$. 
An internal control was included to evaluate the DNA extraction efficiency in all the tissue samples. For this, samples were spiked with $0.32 \mathrm{ng}$ of plasmid DNA (pCM66) prior to extraction. qPCR with pCM66-specific primers (pCM66_Fw: 5' - CTTGGCCCTCACTGACAG-3' and pCM66_Rev, 5' - GCAGCCCTGGTTAAAAAC-3') was performed on a selection of samples to control the extraction efficiency.

qPCR was performed in a $20-\mu \mathrm{l}$ reaction mixture volume containing $10 \mu \mathrm{l}$ of $2 \times \mathrm{iQ}^{\mathrm{m}}$ SYBR green supermix, $0.3 \mu \mathrm{M}$ of each primer, template (genomic DNA, standard) and RNase-free sterile water to a final volume of $20 \mu \mathrm{l}$. The amount of fluorescence generated was measured during each amplification cycle using the following program: (i) initial denaturing at $95{ }^{\circ} \mathrm{C}$ for $10 \mathrm{~min}$; (ii) 40 cycles, with 1 cycle consisting of denaturation at $95^{\circ}$ $\mathrm{C}$ for $10 \mathrm{~s}$, annealing at $60{ }^{\circ} \mathrm{C}$ for $10 \mathrm{~s}$, and extension at $72{ }^{\circ} \mathrm{C}$ for $30 \mathrm{~s}$. All assays were carried out on a LightCycler $^{\mathrm{Tm}}$ (Roche Diagnostics, Mannheim, Germany) in 96-well format plates in duplicate and were averaged for each sample. For each PCR run, a negative (no-template) control was used to test for false-positive results or contamination.

\section{Additional file}

Additional file 1: Figure S1. Number of WT Sodalis CFU in abdomen, thorax and reproductive tissues of streptozotocin-treated (red circle) and non-treated (black $\mathbf{\square})$ female flies. Prior to injection, treated flies were given three blood meals supplemented with $20 \mu \mathrm{g} / \mathrm{ml}$ streptozotocin during the first week after emergence, while non-treated flies received normal blood meals. Data points represent the mean Sodalis CFU $( \pm$ SD) present in the different tissues of at least 5 individual flies at the time of sampling; 4 days post-eclosion. The number of CFU is represented in log scale on the $y$-axis. (DOCX $19 \mathrm{~kb})$

\section{Abbreviations}

CFU: Colony forming unit; GFP: Green fluorescent protein; RecSodalis: GFPtagged Sodalis; SIT: Sterile insect technique; WT: Wild type

\section{Acknowledgements}

We wish to acknowledge the technical assistance of Jos Van Hees.

\section{Funding}

This work was supported by an ITM SOFI grant and the ERC-Starting Grant 'NANOSYM' (282312). This work is also performed in the frame of a FAO/IAEA Coordinated Research Project on "Improving SIT for tsetse flies through research on their symbionts and pathogens" and the publication fee was funded by this project. The funding bodies had no role in the design of the study and collection, analysis, and interpretation of data and in writing the manuscript.

\section{Availability of data and materials}

The datasets used and/or analysed during the current study are available from the corresponding author on reasonable request.

\section{About this supplement}

This article has been published as part of BMC Microbiology Volume 18 Supplement 1, 2018: Enhancing Vector Refractoriness to Trypanosome Infection. The full contents of the supplement are available online at https://bmcmicrobiol.biomedcentral.com/articles/supplements/volume-18supplement-1.

\section{Authors' contributions}

Conceived and designed the experiments: LDV, JVDA. Performed the experiments: LDV, SVK. Analyzed the data: LDV, SVK. Wrote the paper: LDV, SVK, JVDA. All authors read and approved the final manuscript.

\section{Authors' information}

Severien Van Keer currently holds a position as a PhD student at the Centre for the Evaluation of Vaccination, Vaccine and Infectious Disease Institute, University of Antwerp, Antwerpen, Belgium.

Ethics approval and consent to participate

Not applicable.

\section{Consent for publication}

Not applicable.

\section{Competing interests}

The authors declare that they have no competing interests.

\section{Publisher's Note}

Springer Nature remains neutral with regard to jurisdictional claims in published maps and institutional affiliations.

Published: 23 November 2018

References

1. Fevre EM, Picozzi K, Jannin J, Welburn SC, Maudlin I. Human African trypanosomiasis: epidemiology and control. Adv Parasitol. 2013;61:167-221.

2. Brun R, Blum J, Chappuis F, Burri C. Human African trypanosomiasis. Lancet. 2010;375:148-59.

3. Vreysen MJ, Saleh KM, Ali MY, Abdulla AM, Zhu ZR, Juma KG, et al. Glossina austeni (Diptera: Glossinidae) eradicated on the island of Unguja, Zanzibar, using the sterile insect technique. J Econ Entomol. 2000;93:123-35.

4. Balmand S, Lohs C, Aksoy S, Heddi A. Tissue distribution and transmission routes for the tsetse fly endosymbionts. J Invertebr Pathol. 2013;112(Suppl): S116-22.

5. Cheng Q, Aksoy S. Tissue tropism, transmission and expression of foreign genes in vivo in midgut symbionts of tsetse flies. Insect Mol Biol. 1999;8: 125-32.

6. De Vooght L, Caljon G, Stijlemans B, De Beatselier P, Coosemans M, Van Den Abbeele J. Expression and extracellular release of a functional antitrypanosome Nanobody ${ }^{\oplus}$ in Sodalis glossinidius, a bacterial symbiont of the tsetse fly. Microb Cell Factories. 2012;11:23.

7. De Vooght L, Caljon G, De Ridder K, Van Den Abbeele J. Delivery of a functional anti-trypanosome Nanobody in different tsetse fly tissues via a bacterial symbiont, Sodalis glossinidius. Microb Cell Factories. 2014;13:156.

8. De Vooght L, Caljon G, Van Hees J, Van Den Abbeele J. Paternal transmission of a secondary symbiont during mating in the viviparous tsetse fly. Mol Biol Evol. 2015;32:1977-80.

9. Maltz MA, Weiss BL, O'Neill M, Wu Y, Aksoy S. OmpA-mediated biofilm formation is essential for the commensal bacterium Sodalis glossinidius to colonize the tsetse fly gut. Appl Environ Microbiol. 2012;78:7760-8.

10. Attardo GM, Lohs C, Heddi A, Alam UH, Yildirim S, Aksoy S. Analysis of milk gland structure and function in Glossina morsitans: milk protein production, symbiont populations and fecundity. J Insect Physiol. 2008;54:1236-42.

11. Dale C, Young SA, Haydon DT, Welburn SC. The insect endosymbiont S. glossinidius utilizes a type III secretion system for cell invasion. PNAS. 2001; 98:1883-8.

12. Toh H, Weiss BL, Perkin SA, Yamashita A, Oshima K, Hattori M, et al. Massive genome erosion and functional adaptations provide insights into the symbiotic lifestyle of Sodalis glossinidius in the tsetse host. Genome Res. 2006;16:149-56

13. McKenzie GJ, Craig NL. Fast, easy and efficient: site-specific insertion of transgenes into enterobacterial chromosomes using Tn7 without need for selection of the insertion event. BMC Microbiol. 2006;6:39. 